\title{
Potencial miogênico evocado vestibular e suas implicações no domínio das frequências
}

\section{Vestibular evoked myogenic potential and its implications in the frequency domain}

\author{
Aline Tenório Lins Carnaúba', Otávio Gomes Lins², Ilka do Amaral Soares³, Kelly Cristina Lira de Andrade', \\ Pedro de Lemos Menezes ${ }^{4}$
}

\section{RESUMO}

Objetivo: Verificar a existência de associação entre o VEMP no domínio do tempo e no domínio das frequências. Métodos: A amostra foi composta por 18 indivíduos (36 orelhas), sendo seis do gênero masculino e 12 do gênero feminino. No exame de VEMP no domínio do tempo, foram promediados 200 estímulos tone burst na frequência de $500 \mathrm{~Hz}$, com taxa de estimulação de 5,1 estímulos/s na intensidade de 95 dBNAn. Seguiu-se com a captação no domínio das frequências, onde foram promediados 200 estímulos constituídos por tons puros de $500 \mathrm{~Hz}$ (frequência portadora), modulados na frequência de $40 \mathrm{~Hz}$. Foi realizado o cálculo do Odds ratio e aplicado o teste Qui-quadrado para a comparação entre as respostas dos dois domínios. Estudo de coorte contemporânea com corte transversal. Resultados: O VEMP no domínio do tempo foi registrado por meio da estimulação e captação unilateral, com morfologia adequada, em $88,88 \%$ das orelhas. Na realização do exame no domínio das frequências, foi registrada a presença de pico de estado estável em 30 (83,33\%) dos 36 exames. Quando realizado o teste Qui-quadrado e o cálculo do Odds ratio, foi possível observar a existência de forte relação entre os dois domínios. Conclusão: Conclui-se que existe forte associação entre os exames de VEMP no domínio do tempo e no domínio das frequências, o que habilita sua utilização na prática clínica.

Descritores: Testes de Função Vestibular; Potencial Evocado Motor; Estimulação Acústica; Sáculo e Utrículo; Vestíbulo do labirinto

\begin{abstract}
Purpose: Determine whether there is an association between VEMP in the time domain and the frequency domain. Methods: The sample was composed of 18 individuals ( 36 ears), six men and 12 women. In the VEMP test in the time domain, 200 tone burst stimuli were promediated at a frequency of $500 \mathrm{~Hz}$, with 5.1 stimulations/s at an intensity of 95 dBNAn. This was followed by capture in the frequency domain, where 200 stimuli consisting of $500 \mathrm{~Hz}$ (carrier frequency) pure tones were promediated and modulated at a frequency of $40 \mathrm{~Hz}$. Odds ratio was calculated and the chi-squared test was applied to compare the responses of two domains. This is a cross-sectional contemporary cohort study. Results: VEMP in the time domain was recorded by unilateral stimulation and capture, with adequate morphology, in $88.88 \%$ of the ears. In the frequency domain test, a steady-state peak was found in 30 of the 36 tests $(83.33 \%)$. The chi-squared test and odds ratio calculation showed a strong relationship between the two domains. Conclusion: There is a significant association between the VEMP tests in the time and frequency domains, a finding that suppports its use in clinical practice.
\end{abstract}

Keywords: Vestibular Function Tests; Evoked Potentials, Motor; Acoustic Stimulation; Saccule and Utricle; Vestibule, Labyrinth

Trabalho realizado no Programa de Pós-graduação em Saúde da Comunicação Humana da Universidade Federal de Pernambuco - UFPE - Recife (PE), Brasil, com Bolsa REUNI de assistência ao ensino UFPE.

(1) Programa de Pós-graduação (Mestrado) em Saúde da Comunicação Humana, Universidade Federal de Pernambuco - UFPE - Recife (PE), Brasil.

(2) Departamento de Neurologia, Universidade Federal de Pernambuco - UFPE - Recife (PE), Brasil.

(3) Programa de Pós-graduação (Doutorado) em Neuropsiquiatria e Ciência do Comportamento, Universidade Federal de Pernambuco - UFPE - Recife (PE), Brasil.

(4) Departamento de Fonoaudiologia, Universidade Estadual de Ciências da Saúde de Alagoas - UNCISAL - Maceió (AL), Brasil.

Conflito de interesses: Não

Contribuição dos autores: $A T L C$ pesquisadora principal, elaboração da pesquisa, elaboração do cronograma, levantamento da literatura, coleta e análise dos dados, redação do artigo, submissão e trâmites do artigo; $O G L$ coorientador, elaboração da pesquisa, elaboração do cronograma, análise dos dados, correção da redação do artigo; IAS coorientadora, elaboração da pesquisa, elaboração do cronograma, análise dos dados, correção da redação do artigo, aprovação da versão final; KCLA pesquisadora, levantamento da literatura, coleta e análise dos dados, redação do artigo; PLM orientador, elaboração da pesquisa, elaboração do cronograma, análise dos dados, correção da redação do artigo, aprovação da versão final.

Endereço para correspondência: Aline Tenório Lins Carnaúba. Programa de Pós-graduação em Saúde da Comunicação Humana. R. Arthur de Sá, s/n, Cidade Universitária, Recife (PE), Brasil, CEP: 50670-420. E-mail: alinel.tenorio@ gmail.com

Recebido em: 17/3/2013; Aceito em: 4/9/2013 


\section{INTRODUÇÃO}

Desde a sua primeira descrição em $1992^{(1)}$, o potencial miogênico evocado vestibular (VEMP) tornou-se um teste complementar da função vestibular. O VEMP é um reflexo muscular, evocado pela estimulação da via vestibulo cervical (normalmente com som alto) ${ }^{(2-4)}$ e registrado a partir de eletrodos colocados sobre o músculo esternocleidomastoideo ${ }^{(5)}$.

Por meio desse exame, avalia-se a integridade do sáculo e do nervo vestibular inferior, informações não obtidas por meio da avaliação vestibular tradicional, como a vectoeletronistagmografia ${ }^{(6) .}$

As ondas do VEMP são altamente reprodutíveis, independente da estimulação do esternocleidomastoideo ser unilateral ou bilateral, ou da captação ser realizada em momentos diferentes. Em decorrência disso, o VEMP pode ser utilizado clinicamente, com inúmeras aplicações no diagnóstico das desordens vestibulares ${ }^{(7)}$.

Tradicionalmente, os sinais biológicos do VEMP são analisados no domínio do tempo. Na maioria das vezes, o registro é feito sob forma de onda, que representa a variação do potencial elétrico no tempo. Entretanto, artefatos e anormalidades do sinal, nesse domínio, podem dificultar a interpretação e o exame depende exclusivamente do conhecimento e da experiência do examinador. Com o objetivo de extrair as informações mais relevantes e eliminar as interferências, podem ser empregadas técnicas de processamento de sinais, como, por exemplo, a Transformada de Fourier, que observa o evento não mais no domínio do tempo, mas no domínio das frequências ${ }^{(8)}$.

A promissora técnica de estado estável avalia os potenciais auditivos no domínio das frequências. Tons modulados podem ser apresentados às duas orelhas, simultaneamente, e a resposta analisada por meio da Transformada Rápida de Fourier, associada a técnicas estatísticas ${ }^{(9,10)}$. É um exame muito mais rápido e preciso, quanto ao comprometimento das frequências ${ }^{(11)}$.

Estudos realizados sobre a utilização da técnica de estado estável para a detecção do VEMP, referem que esse exame, assim como o potencial evocado auditivo, também pode ser realizado no domínio das frequências ${ }^{(7,12)}$. Além de possibilitar a detecção de acometimentos do nervo vestibular inferior em estágios iniciais, favorece o diagnóstico mais preciso, em um intervalo de tempo menor e independente da interpretação do avaliador ${ }^{(7)}$.

No domínio do tempo, a resposta é registrada e analisada pela seleção dos picos e avaliação das amplitudes e latências. No domínio da frequência, a análise baseia-se na amplitude e fase da resposta de cada pico do espectro de frequência ${ }^{(13,14)}$.

Sendo assim, o objetivo deste estudo foi verificar a existência de associação entre o potencial miogênico evocado vestibular no domínio do tempo e no domínio das frequências.

\section{MÉTODOS}

O estudo foi aprovado pelo Comitê de Ética em Pesquisa da Universidade Estadual de Ciências da Saúde de Alagoas (UNCISAL), mediante o parecer $n^{\circ} 990 / 09$. Todos os participantes assinaram o Termo de Consentimento Livre e Esclarecido.

A pesquisa foi divulgada aos professores, alunos e funcionários de universidades públicas e privadas da cidade de Maceió, Alagoas. Os sujeitos compareceram, espontaneamente, ao local do estudo e não obtiveram nenhuma contribuição financeira para a realização do experimento. Receberam, entretanto, cópia de toda a avaliação auditiva e vestibular realizada.

Fizeram parte do estudo 18 indivíduos (36 orelhas), sendo seis do gênero masculino e 12 do gênero feminino, selecionados considerando-se os seguintes critérios de inclusão: idades entre 18 e 35 anos e limiares auditivos iguais ou inferiores a 20 dBNA, com diferenças entre as orelhas iguais ou inferiores a 10 $\mathrm{dB}$, por frequência. Foram excluídos aqueles que apresentavam exposição a ruído ocupacional ou de lazer, cirurgias na orelha média e/ou interna, mais de três infecções de orelha média, uso de medicação ototóxica, presença de alterações sistêmicas, tais como diabetes e hipertensão arterial, que pudessem contribuir para patologias cócleo-vestibulares, alterações hormonais e presença de zumbido, vertigens, tonturas, ou outras alterações cócleo-vestibulares.

Inicialmente, foi aplicado um questionário sobre a história de saúde geral e da função auditiva e vestibular, para a triagem dos participantes (Anexo 1). Logo após, foram realizados os seguintes procedimentos: otoscopia, audiometria tonal liminar e VEMP no domínio do tempo e no domínio das frequências.

Os exames de VEMP foram realizados com um aparelho específico, o Analisador de potenciais evocados no domínio do tempo e das frequências (APEDTF). Desenvolvido em duas universidades públicas, o APEDTF é composto por amplificadores biológicos, filtros, sistema de proteção elétrica e um sistema lógico que possibilita a investigação do VEMP.

Para o registro dos potenciais, foram utilizados eletrodos de superfície, colocados sobre a pele previamente limpa, posicionando-se o eletrodo positivo no terço médio do músculo esternocleidomastoideo (ECM) ipsilateral à estimulação. $\mathrm{O}$ eletrodo negativo foi posicionado na altura do tendão do músculo, logo acima da clavícula, e o eletrodo terra, na linha média frontal. Para obtenção do registro no músculo ECM, o paciente permaneceu sentado, com rotação lateral máxima de cabeça para o lado contralateral ao estímulo.

No exame de VEMP no domínio do tempo, foram promediados 200 estímulos tone burst na frequência de $500 \mathrm{~Hz}$, taxa de estimulação em 40,8 estímulos/s na intensidade de 95 dBNAn, apresentados por meio de fones de inserção ER 3A, com olivas de espuma descartáveis. Foi utilizado um filtro "passa banda", configurado entre 5 e $1000 \mathrm{~Hz}$, com exibição de 10 a $25 \mu \mathrm{V}$, por divisão. Os registros foram realizados em janelas de $40 \mathrm{~ms}$, por apresentarem tempo suficiente para englobar todas as respostas ${ }^{(15,16)}$.

Para a interpretação dos achados, as respostas foram 
Tabela 1. Latências e amplitudes do VEMP no domínio do tempo

\begin{tabular}{llcccc}
\hline & & \multicolumn{2}{c}{ Latência $(\mathrm{ms})$} & \multicolumn{3}{c}{ Amplitude $(\mu \mathrm{V})$} \\
\cline { 3 - 6 } & & $\mathrm{p} 13$ & $\mathrm{n} 23$ & $\mathrm{p} 13$ & $\mathrm{n}$ \\
\hline \multirow{2}{*}{40,8 estímulos $/ \mathrm{s}$} & Média & 14,84 & 24,11 & 27,92 & $-23,42$ \\
& DP & 2,71 & 4,12 & 2,15 & 2,57 \\
\hline
\end{tabular}

Legenda: VEMP = potencial miogênico evocado vestibular; $\mathrm{DP}=$ desvio-padrão

analisadas por dois autores/avaliadores, por meio da morfologia, demarcando-se as ondas p13 e n23 pelas latências dos primeiros picos positivo e negativo. As discrepâncias apresentadas pelos autores/avaliadores foram esclarecidas por um terceiro autor/avaliador.

Para captação do VEMP no domínio das frequências, foram promediados 200 estímulos constituídos por tons puros de 500 $\mathrm{Hz}$ (frequência portadora), modulados em $90 \%$ de suas potências de amplitude, na frequência de $40 \mathrm{~Hz}$, apresentados por meio de fones de inserção ER 3A. Os registros foram realizados em janelas de $100 \mathrm{~Hz}$.

Para a análise do VEMP no domínio das frequências, as potências das frequências adquiridas pela Transformada Rápida de Fourier foram promediadas 200 vezes e analisadas, posteriormente, pelo método estatístico da coerência de magnitude quadrada (CMQ), para que as respostas fossem confirmadas.

A escolha da moduladora para o exame no domínio das frequências baseou-se nas repostas obtidas na avaliação dos potenciais evocados auditivos de estado estável. Para esse exame, a taxa de modulação de $40 \mathrm{~Hz}$ é a que apresenta respostas com ondas de maior amplitude ${ }^{(17)}$. Além disso, essa frequência de modulação equivale a uma observação de $25 \mathrm{~ms}$ no domínio do tempo, o que é apropriado para avaliar o VEMP, que possui componente de onda mais tardio próximo a $23 \mathrm{~ms}^{(6)}$.

Na realização do VEMP no domínio das frequências, o equipamento apresentou as seguintes características fundamentais para o processamento desse tipo de sinal: conversão dos sinais digital e analógico, realizada a uma taxa de amostragem de $44,1 \mathrm{kHz}$ e resolução de 16 bits; conversão analógica digital, conduzida com frequência de aquisição de $2,75 \mathrm{kHz}$, exatamente 1/16 da taxa de geração do sinal, para garantir a Transformada Rápida de Fourier de até 1,3 kHz, também com resolução de 16 bits.

\section{Método estatístico}

Os dados foram tabulados e processados pelo aplicativo para microcomputador Predictive Analytics Software (PASW® STATISTIC), versão 17.0. Para a descrição dos dados, utilizou-se a apresentação tabular e gráfica das médias dos desvios-padrão e dos percentis.

Para comprovar se os percentuais de presença de resposta entre os domínios foram estatisticamente semelhantes, aplicou-se o cálculo do risco Odds ratio e o teste Qui-quadrado.

\section{RESULTADOS}

O VEMP no domínio do tempo foi registrado por meio da estimulação e captação unilateral, com morfologia adequada, em $88,88 \%$ das orelhas, utilizando-se a taxa de estimulação de 40,8 estímulos/s, com estímulo tone burst na frequência de $500 \mathrm{~Hz}$.

No traçado do exame, foi realizada marcação das ondas, determinando-se as latências e amplitudes absolutas de p13 e n23 (Tabela 1).

Na realização do exame no domínio das frequências, foi registrada a presença de pico de estado estável em 30 (83,33\%) dos 36 exames realizados. Dessa amostra, uma pessoa (dois exames) apresentou respostas apenas no domínio do tempo e outras duas pessoas (quatro exames), não apresentaram respostas em nenhum dos domínios avaliados.

O teste Qui-quadrado, realizado para comparar as presenças e ausências de respostas entre os dois domínios, comprovou que as pequenas diferenças numéricas foram significativas, ou seja, quando as respostas estavam presentes no domínio do tempo, também estavam presentes no domínio das frequências (Tabelas 2 e 3).

Tabela 2. Relação entre presença do pico p13 do VEMP no domínio do tempo com a presença do pico do VEMP no domínio das frequências

\begin{tabular}{lccc}
\hline & \multicolumn{3}{c}{ Presença do pico do VEMP no DF } \\
\cline { 2 - 4 } & Presente & Ausente & Total \\
\hline Presença do pico p13 & 27 & 2 & 29 \\
Presente & 3 & 4 & 7 \\
Ausente & 30 & 6 & 36 \\
Total & & $0,008^{*}$ & \\
Valor de $\mathrm{p}$ & &
\end{tabular}

${ }^{*}$ Valores significativos $(p \leq 0,05)$ - Teste Qui-quadrado

Legenda: VEMP = potencial miogênico evocado vestibular; DF = domínio das frequências

Tabela 3. Relação entre presença do pico n23 do VEMP no domínio do tempo com a presença do pico do VEMP no domínio das frequências

\begin{tabular}{lccc}
\hline & \multicolumn{3}{c}{ Presença do pico do VEMP no DF } \\
\cline { 2 - 4 } & Presente & Ausente & Total \\
\hline Presença do pico n23 & 25 & 2 & 27 \\
Presente & 5 & 4 & 9 \\
Ausente & 30 & 6 & 36 \\
Total & & $0,024^{*}$ & \\
Valor de $p$ & &
\end{tabular}

*Valores significativos $(p \leq 0,05)$ - Teste Qui-quadrado

Legenda: $\mathrm{VEMP}$ = potencial miogênico evocado vestibular; DF = domínio das frequências 
Tabela 4. Investigação sobre associação entre presença de onda no domínio do tempo e no domínio das frequências do exame de VEMP

\begin{tabular}{|c|c|c|c|c|c|c|c|}
\hline \multirow{2}{*}{$\begin{array}{l}\text { Frequência } \\
\text { moduladora }\end{array}$} & \multirow{2}{*}{ VEMP DT } & \multirow{2}{*}{$\begin{array}{c}\text { Associação entre } \\
\text { pico e onda }\end{array}$} & \multicolumn{2}{|c|}{ Igualdade } & \multirow{2}{*}{ Odds ratio } & \multicolumn{2}{|c|}{ Intervalo de confiança $(95 \%$} \\
\hline & & & $\operatorname{Sim}$ & Não & & Inferior & Superior \\
\hline \multirow[t]{2}{*}{$40 \mathrm{~Hz}$} & p13 & $86,11 \%$ & 31 & 5 & 18 & 2,26 & 143,3 \\
\hline & $\mathrm{n} 23$ & $80,50 \%$ & 29 & 7 & 10 & 1,42 & 70,29 \\
\hline
\end{tabular}

Legenda: VEMP = potencial miogênico evocado vestibular; $\mathrm{DT}$ = domínio do tempo

Quando realizado o cálculo do Odds ratio, foi constatada associação entre presença das ondas p13 e n23 de VEMP no domínio do tempo e presença de pico de estado estável do VEMP no domínio das frequências, com valor de Odds ratio igual a 18 e 10, respectivamente, ou seja, na presença de onda no tempo (p13), existem 18 vezes mais chances de também existir presença de pico no exame de estado estável, como também, na presença da onda $\mathrm{n} 23$, existem dez vezes mais chances de existir o pico de estado estável (Tabela 4).

\section{DISCUSSÃO}

A análise das respostas obtidas nesta amostra demonstrou que é possível registrar de forma consistente as ondas p13 e n23 no domínio do tempo, apresentando resultados similares a outros estudos ${ }^{(15,18)}$.

Com relação à ausência de respostas nos dois domínios, em quatro exames realizados, podemos estabelecer duas hipóteses: a primeira é que os dois sujeitos em questão tenham apresentado alteração vestibular em alguma parte da trajetória do reflexo vestíbulo cervical ${ }^{(16,19)}$; a segunda, leva em conta os fatores externos envolvidos na avaliação do potencial, como presença de artefatos que possam ter causado ausência de respostas, ou alterações no estado de vigília do paciente ${ }^{(9)}$.

No que diz respeito à associação entre os dois domínios, pode-se inferir, com base nos resultados do presente estudo e da literatura, a existência de forte relação entre os dois domínios, confirmando que é possível realizar VEMP no domínio das frequências, sem perder as informações necessárias para avaliação vestibular ${ }^{(10,11)}$.

Estabelecendo-se uma analogia com o Potencial evocado auditivo de estado estável (PEAEE), a grande vantagem do VEMP no domínio das frequências está na forma objetiva de avaliação das respostas, que pode ser feita automaticamente, por meio de softwares. Isso diminui a probabilidade de erros de diagnóstico, sobretudo com intensidades um pouco menores, uma vez que não é necessária a interpretação subjetiva de um profissional por meio da avaliação visual dos resultados ${ }^{(8,9)}$. Além disso, a utilização dessa técnica não requer que os pacientes estejam sedados e oferece a possibilidade de exploração simultânea de várias frequências, sem aumento significativo no tempo de avaliação ${ }^{(9,13)}$.

Alguns autores relatam que a utilização do VEMP no domínio do tempo, em pessoas com audição normal, traz uma limitação ao uso clinico, devido à exposição sonora elevada durante a realização do teste ${ }^{(11,20)}$. Outra contraindicação, seria a de que individuos com queixas de zumbido não poderiam ser submetidos ao teste, pois implicaria na piora dos sintomas ${ }^{(11)}$. No entanto, o VEMP no domínio das frequências, por ser um exame mais rápido, minimiza essas contraindicações e, além disso, pode avaliar várias frequências simultaneamente, o que demonstra sua utilidade para avaliar a função sacular, possibilitando o diagnóstivo precoce da Doença de Ménière ${ }^{(21)}$.

Por fim, na avaliação do VEMP, os picos p13 e n23 não representam a passagem do potencial por estruturas da via vestibular, como ocorre no potencial auditivo de tronco encefálico (PEATE) com as ondas de I a V, na via correspondente ${ }^{(16)}$. Assim, o VEMP no domínio das frequências não perde informações temporais, que auxiliariam em possíveis identificações da origem da interrupção da transmissão nervosa ${ }^{(21)}$.

\section{CONCLUSÃO}

Houve forte associação entre os exames de VEMP no domínio do tempo e no domínio das frequências, o que habilita sua utilização na prática clínica.

\section{REFERÊNCIAS}

1. Colebatch JG, Halmagyi GM. Vestibular evoked potentials in human neck muscles before and after unilateral vestibular deafferentation. Neurology.1992;42(8):1635-36.

2. Rosengren SM, Kingma H. New perspectives on vestibular evoked myogenic Potentials. Curr Opin Neurol. 2013;26(1):74-80.

3. Rocha MF, Azevedo DF, Russomano T, Figueira MV, Helegda S. Mobile remote monitoring of biological signals. In: IEEE 2006 International Conference of the Engineering in Medicine and Biology Society, 2006, Nova York. IEEE 2006 International Conference of the Engineering in Medicine and Biology Society. NY, EUA: IEEE-EMBS, 2006;1:2057-59.

4. Rauch SD. Vestibular evoked myogenic potentials. Otol Neurotol. 2006;14(5):299-304.

5. Sazgar AA, Akrami K, Akrami S, Yazdi ARK. Recording of vestibular evoked myogenic potentials. Acta Med Iran. 2006;44(1):13-6.

6. Hall JW. New Handbook for auditory evoked responses. Boston: Pearson Education. 2006.

7. Barreto ACO, Colafêmina JF, Menezes PL. Saccular sensitivity function measured by vestibular evoked myogenic potential. Acta Otolaryngol. 2011;131(6):618-23. 
8. Akin FW, Murnane OD, Panus PC, Caruthers SK, Wilkinson AE, Proffitt TM. The influence of voluntary tonic EMG level on the vestibular-evoked myogenic potential. J Rehabil Res Dev. 2004;41(3B):473-80.

9. Ramos EG, Zaeyen EJB, Simpsoni DM, Infantosi AFC. Detecção da resposta auditiva no EEG de crianças utilizando técnicas no domínio da frequência. Rev Bras Eng Biomed. 2000;16(3):127-37.

10. Menezes PL. Desenvolvimento e avaliação de um dispositivo capaz de registrar e analisar potenciais evocados auditivos no domínio do tempo e no domínio das frequências por meio de uma placa de som para computador pessoal [tese]. Ribeirão Preto: Universidade de São Paulo, Departamento de Física e Matemática; 2008.

11. Lins OG. Audiometria fisiológica tonal utilizando respostas de estado estável auditivas do tronco cerebral [tese]. São Paulo: Universidade Federal de São Paulo - Escola Paulista de Medicina, Departamento de Medicina; 2002.

12. Bell SL, Fox L, Id Bihi R. Vestibular evoked myogenic responses to amplitude modulated sounds (L). J Acoust Soc Am. 2010;128(2):559-62.

13. Stapells DR, Herdman A, Small SA, Dimitrijevic A, Hatton J. Current status of the auditory steady-state responser for estimating an infant`s audiogram. In: Seewald RC, Banckford J. (Eds.) A Sound Foundation Through Early Amplification. Chicago: Phonak, 2005. p. 43-59.
14. Picton TW, John MS, Dimitrijevic A, Purcell D. Human auditory steady-state response. Int J Audiol. 2003;42(4):177-219.

15. Basta D, Todt I, Ernst A. Normative data for P1/N1 latencies of vestibular evoked myogenic potencials induced by air or bone conducted tone bursts. Clin Neurophysiol. 2005;116(9):2216-9.

16. Sheykholeslami K, Habiby Kermany M, Kaga K. Frequency sensitivity range of the saccule to bone conducted stimuli measured by vestibular evoked myogenic potencials. Hear Res. 2001;160(12):58-62.

17. Dimitrijevic A, John MS, Picton TW. Auditory steady-state responses and word recognition scores in normal hearing and hearing impaired adults. Ear Hear. 2004;25(1):68-84.

18. Wu CH, Murofushi T. The effect of click repetition rate on vestibular evoked myogenic potenctial. Acta Otolaryngol. 1999;119(1):29-32.

19. Sheykholeslami K, Kermany MH, Kaga K, Megerian CA, Arnold J. Vestibular-evoked myogenic potencials in infancy and early childhood. Laryngoscope. 2005;115(8):1440-4.

20. Rauch SD, Silveira MB, Zhou G, Kujawa SG, Wall C $3^{\text {rd }}$, Guinan JJ, et al. Vestibular evoked myogenic potentials versus vestibular test battery in battery in patients with Menière's disease. Otol. Neurotol. 2004;25(6):981-6.

21. Rosengren SM, Welgampola MS, Colebatch JG. Vestibular evoked myogenic potentials: past, present and future. Clin Neurophysiol. 2010;121(5):636-51.

Anexo 1. Questionário sobre a história de saúde geral e da função auditiva e vestibular

Identificação

Nome:

Data: Idade: Gênero:

\section{Passado otológico}

Infecções de ouvido (mais de três episódios/ano): S ( ) N ( )

Uso de medicação ototóxica: $\mathrm{S}(\mathrm{)}) \mathrm{N}(\mathrm{)}$

Cirurgias de ouvido: S ( ) N ( ) Tipo:

Presença de zumbido, vertigens, tonturas ou outras alterações cócleo-vestibulares: S ( ) N ( )

Hereditariedade para surdez: . S ( ) $\mathrm{N}($ )

Exposição a ruído ocupacional ou de lazer:......... S ( ) N ( )

Em caso afirmativo, tempo mínimo semanal:

Repouso auditivo mínimo de 14 horas: $S() \mathrm{N}()$

Estado de saúde geral

Doenças:

Medicações:

Alterações hormonais: S( ) N( )

Inspeção do conduto auditivo externo...

( ) Normal ( ) Alterada

$\mathrm{N}^{\circ}$ do protocolo: 